\title{
Parâmetros genéticos e ganho com a seleção para populações de pinhão manso (Jatropha curcas)
}

\author{
Ana Cristina Pinto Juhász ${ }^{(1)}$, Danielle de Lourdes Batista Morais ${ }^{(2)}$, Bruno Oliveira Soares ${ }^{(3)}$, Samy Pimenta ${ }^{(3)}$, \\ Hudson de Oliveira Rabello(3), Marcos Deon Vilela de Resende ${ }^{(4)}$
}

(1) Empresa de Pesquisa Agropecuária de Minas Gerais, Unidade Regional do Triângulo e Alto Paranaíba, CEP 38001-970, Uberaba, MG., anacpj@yahoo.com.br; (2) Biojan, Rua Américo Soares, 670, CEP 39440-000, Janaúba, MG, moraisdlb@yahoo.com.br; (3) Universidade Estadual de Montes Claros, Campus Janaúba, CEP 39440-000, Montes Claros, MG, brunoosoares@yahoo.com.br; samypimenta@bol.com.br; hudsonorabelo@gmail.com; ${ }^{(4)}$ Embrapa Florestas, Estrada da Ribeira, Km 111, CP 319, CEP 83411-000, Colombo, PR, deon@cnpf.embrapa.br

Resumo - O presente estudo objetivou estimar parâmetros genéticos da fase vegetativa em cinco populações de pinhão manso (Jatropha curcas L.). As progênies de meios-irmãos foram plantadas na Fazenda da Biojan, em Janaúba, MG. Aos três e seis meses de idade, foram avaliadas as características altura de plantas, diâmetro de caule, número de ramos e projeção da copa, em todas as plantas. A população 2 não apresentou variabilidade genética para os caracteres avaliados. Nas demais populações, com a seleção dos dez melhores indivíduos, os ganhos genéticos preditos variaram de $4 \%$ a $8 \%$ para altura de plantas (populações 1 e 4), $5 \%$ a $7 \%$ para diâmetro de caule (populações 1 e 5), $14 \%$ e $25 \%$ para número de ramos aos 3 meses (populações 3 e 5 ) e $9 \%$ para projeção da copa na população 4. De acordo com os resultados observados neste trabalho, estas populações são adequadas para dar continuidade ao programa de melhoramento genético da espécie na região.

Termos para indexação: Variabilidade, herdabilidade, acurácia, ganho genético.

\section{Genetic evaluation and genetic gains with the selection for Jatropha curcas populations}

\begin{abstract}
This work aimed at estimating genetic parameters from the vegetative stage in five populations from Jatropha curcas L. The half sib progenies were cultivated at Biojan farm, in Janaúba, MG. Plant height, stem diameter, number of branches and crown projection were assessed in all plants at three and six months old. In population 2, there was no genetic variability for all traits evaluated. The genetic gains estimated with the selection from the ten best individuals in other populations were $4 \%$ to $8 \%$ for plant height (population 1 and 4 ), $5 \%$ to $7 \%$ for stem diameter (population 1 and 5), $14 \%$ to $25 \%$ for the number or branches (population 3 and 5 ) and $9 \%$ for crown projections in population 4 . According to the results obtained from this study, these populations are adequate for the continuity of the breeding program of this species in the region.
\end{abstract}

Index terms: Variability, heritability, accuracy, genetic gain.

\section{Introdução}

Jatropha curcas, conhecido popularmente como pinhão manso, é uma espécie nativa do Brasil, México, Caribe e América Central (Heller, 1996). Pertence à família das Euphorbiaceaes. Trata-se de uma espécie exigente em insolação e com forte resistência à seca. Configura-se em uma alternativa atraente para produção de óleo para fins energéticos (Arruda et al., 2004), uma vez que o novo interesse global em biocombustíveis vem se expandindo rapidamente. Apesar de o Brasil ter requerido a primeira patente mundial para o biodiesel na década de 1970, o país ainda está muito atrasado em relação a outros países. Tornou-se obrigatório a adição de $2 \%$ de biodiesel no óleo diesel vendido no país a partir de 2008, sendo que era previsto para 2010, um aumento no percentual para 5\%, o que exigirá a produção interna de mais de 2 bilhões de litros de biodiesel por ano, podendo alcançar $20 \%$ da mistura, gerando uma economia de óleo diesel de mais de 9 bilhões de litros por ano (Bsbios, 2008).

De acordo com Resende (2002), as espécies vegetais perenes apresentam vários aspectos biológicos peculiares, que tornam o seu melhoramento genético bastante diferenciado do melhoramento de culturas anuais. Dentre eles, citam-se: sobreposição de gerações, 
ciclo reprodutivo longo, reprodução sexuada e assexuada e expressão dos caracteres ao longo das várias idades.

Neste contexto, os testes de progênies, instrumentos importantes para o trabalho do melhorista, têm sido usados na estimação de parâmetros genéticos e seleção de indivíduos, quando se procura avaliar a magnitude e a natureza da variância genética disponível, com vistas a quantificar e maximizar os ganhos genéticos, utilizando-se procedimento de seleção adequado (Costa et al., 2006).

A metodologia de avaliação genética REML/BLUP vem sendo aplicada em experimentos de avaliação de famílias, populações e clones de pinhão manso (Abreu et al., 2009).

Esta metodologia tem sido também utilizada para a seleção em diversas culturas perenes, como em acerola (Paiva et al., 2007) e caju (Cavalcanti et al., 2007), pínus (Missio et al., 2004, 2005), eucalipto (Garcia \& Nogueira, 2005; Rocha et al., 2006), pupunha (Farias Neto \& Resende, 2001), seringueira (Costa et al., 2002a, 2002b), erva-mate (Costa et al., 2006) e cupuaçu (Souza et al., 2002), entre outras.

Como o pinhão manso é uma espécie ainda não domesticada, os trabalhos de melhoramento estão em fase inicial de desenvolvimento e a literatura fornece apenas resultados iniciais relacionados à diversidade genética entre acessos de pinhão, como os desenvolvidos na Índia por Ginwal et al. (2004), Ginwal et al. (2005), Kaushik et al. (2007) e Rao et al. (2008), e no Brasil por Abreu et al. (2009). Desta forma, é necessário um estudo mais detalhado da espécie, tanto na fase vegetativa, como na reprodutiva, para que seja possível estabelecer correlações entre características vegetativas e reprodutivas, assim como ocorre em outras espécies perenes, como, por exemplo, em açaí (Farias Neto et al., 2005) e pupunha (Nogueira et al., 2004). O porte da planta, por exemplo, apresenta correlação favorável com a produção total de óleo por planta. Isto explica a necessidade de considerar estas características nesta fase do melhoramento.

Após a avaliação da fase vegetativa, a reprodutiva é de suma importância, assim como a avaliação do teor de óleo de cada planta avaliada no programa. A partir de parâmetros genéticos relacionados à produção de óleo, o melhorista terá um embasamento para decidir como conduzir futuros programas de melhoramento. No entanto, deve-se ressaltar que esta característica, assim como outras relacionadas à produção, precisam ser avaliadas por alguns anos consecutivos, para se estimar a repetibilidade e verificar em que momento a planta se estabelecerá de forma a manter uma produtividade constante, ou chegar à maturidade reprodutiva.

Diante do exposto, o trabalho teve por objetivo estimar parâmetros genéticos na fase vegetativa de cinco populações de pinhão manso (Jatropha curcas). Os resultados, que incluem a estimativa de ganhos genéticos com a seleção, visam subsidiar o programa de melhoramento genético dessa espécie na região norte de Minas Gerais e também contribuir para o aumento do conhecimento do controle genético de caracteres vegetativos nessa espécie que, num segundo momento, serão correlacionados com características reprodutivas e com o teor de óleo.

\section{Material e métodos}

Foram conduzidas cinco populações em Janaúba, MG, na Fazenda da Biojan, localizada a $15^{\circ} 43^{\prime} 94^{\prime \prime} S$ e $43^{\circ} 19^{\prime} 571^{\prime \prime} \mathrm{W}$, com altitude de $516 \mathrm{~m}$, temperatura média máxima anual de $31{ }^{\circ} \mathrm{C}$, mínima de $21{ }^{\circ} \mathrm{C}$ e pluviosidade média anual de $800 \mathrm{~mm}$. Os experimentos foram conduzidos no período de abril de 2008 a janeiro de 2009.

Este trabalho foi realizado em parceria com a Biojan, empresa produtora de sementes de pinhão manso, em Janaúba, MG. Na Fazenda Biojan, as áreas de produção foram agrupadas em setores, compostos por plantas adultas, em idade superior a dois anos, com algumas características em comum, por exemplo, ramificação e altura de plantas. Desta forma, foram selecionados cinco setores de produção e, de cada um, foram selecionadas dez plantas matrizes superiores para formar as dez progênies de meios irmãos de cada setor (população).

O delineamento adotado para cada experimento foi o de blocos ao acaso, com dez tratamentos (progênies), 40 blocos e uma planta por parcela. As plantas foram dispostas em linhas duplas, com espaçamento de $2 \mathrm{~m}$ entre plantas, $3 \mathrm{~m}$ entre linhas e $6 \mathrm{~m}$ entre linhas duplas, da mesma forma como é cultivado o pinhão na propriedade, facilitando os tratos culturais, como as pulverizações.

As dez plantas de cada progênie foram distribuídas ao acaso em uma linha simples, formando um bloco. $\mathrm{O}$ plantio foi feito por meio da semeadura direta no campo, utilizando-se duas sementes por cova. Após a emissão das primeiras folhas definitivas, foi feito o desbaste, 
permanecendo apenas uma planta por cova. As plantas foram irrigadas por gotejamento.

Aos 3 e 6 meses de idade, as progênies foram avaliadas quanto aos caracteres: a) Altura de plantas - utilizando-se uma régua para medir a altura total de cada planta, em centímetros, compreendida entre a base do caule (solo) e a extremidade do ramo mais alto; b) Diâmetro de caule - avaliado com o auxílio de um paquímetro digital, em milímetros, a uma distância de $10 \mathrm{~cm}$ da base do caule principal; c) Número de ramos por planta - foi contabilizado o número de ramos primários que formam a copa de cada planta. Foram considerados como ramos principais aqueles inseridos no caule principal até uma altura de $40 \mathrm{~cm}$ da base do caule (solo). Previamente à análise, constatou-se a normalidade dos dados, estudando-se a assimetria e a curtose da distribuição e testando-os pelo teste $t$, concluindo pela distribuição simétrica e mesocúrtica; d) Projeção da copa - foi utilizada uma régua para medir o diâmetro da projeção da copa, em centímetros, na posição da entrelinha de plantio.

No total, foram avaliadas 400 plantas de cada população, perfazendo 2 mil plantas com as cinco populações e 16 mil avaliações, uma vez que cada característica foi avaliada duas vezes em cada planta, uma aos 3 meses e a outra 6 meses após o plantio. Em cada população, o tamanho efetivo populacional equivalente foi de 37 , dado por:

$$
N_{e}=\frac{4 N_{f} \bar{k}_{f}}{\bar{k}_{f}+3}
$$

sendo $N_{f}$ o número de famílias e $k_{f}(=40)$ o número de indivíduos por família. Esse tamanho efetivo é adequado para representar uma população, pois permite capturar alelos com frequência maior que $6 \%$ (Resende, 2002). Assim, foram estimados parâmetros genéticos para cada população.

O ganho genético esperado com seleção é estimado pela expressão

$$
\hat{G}_{s}=k \quad r_{\hat{g} g} \sigma_{g}
$$

em que k é o diferencial de seleção padronizado, $r_{\hat{g} g}$ é a acurácia seletiva e $\sigma_{g}^{2}$ é a variância genética disponível na população. Esta expressão permite a comparação de métodos de seleção por meio de um só parâmetro, a acurácia. Isto porque $\sigma_{g}$ é uma propriedade da população e, portanto, constante através dos métodos de seleção. O coeficiente $k$ também pode ser considerado constante através dos métodos de seleção (Resende, 2002).
A acurácia refere-se à correlação entre os valores genéticos preditos e os valores genéticos verdadeiros dos indivíduos. Quanto maior a acurácia na avaliação de um indivíduo, maior é a confiança na avaliação e no valor genético predito deste indivíduo e maior será o ganho com a seleção. Dessa forma, o método ótimo de seleção deve ser aquele que maximiza a acurácia. Esse método é o BLUP individual. O BLUP permite também o uso simultâneo de várias fontes de informação, como aquelas advindas de vários experimentos instalados em um ou vários locais. Para aplicação do BLUP são necessárias estimativas de componentes de variância e de parâmetros genéticos, como as da herdabilidade (Resende, 2002).

O procedimento ótimo de estimação desses componentes de variância é o de máxima verossimilhança residual ou restrita (REML), o qual é superior ao método da análise de variância (ANOVA) em situação de dados desbalanceados e delineamentos não ortogonais (como alguns blocos incompletos). O procedimento ótimo de avaliação genética é, então, o REML/BLUP. Detalhes sobre o REML/BLUP são apresentados por Resende et al. (1993; 1996) e Resende (2002; 2007a, 2007b).

A análise estatística e a estimação de parâmetros genéticos foram baseadas em modelos mistos do tipo REML/BLUP, empregando o software Selegen-REML/ BLUP, conforme Resende (2000, 2002). O modelo estatístico empregado foi dado por $y=X r+Z a+e$, em que $y$ é o vetor de dados, $r$ é o vetor dos efeitos de repetição (assumidos como fixos) somados à média geral, $a$ é o vetor dos efeitos genéticos aditivos individuais (assumidos como aleatórios), $e$ é o vetor de erros ou resíduos (aleatórios). As letras maiúsculas representam as matrizes de incidência para os referidos efeitos. O teste de médias entre as populações para cada característica avaliada foi realizado por meio do teste $t$ de Student, ao nível de significância de 5\%, considerando os desvios padrões das médias de cada população.

Como critério de seleção, adotou-se a seleção univariada com base nos maiores valores genéticos preditos pelo BLUP. A acurácia foi determinada por meio da inversão da matriz dos coeficientes das equações de modelo misto, conforme descrito por Resende (2002).

\section{Resultados e discussão}

Os parâmetros genéticos estimados variaram muito entre as populações, para cada característica avaliada. Não houve uma concordância de resultados, o que indica 
que cada população teve uma base genética diferenciada, refletindo diferenças na composição genética das várias populações.

Para nenhuma das características avaliadas e em nenhuma das populações foram encontrados valores de coeficiente de variação relativa (CVr) acima da unidade. Segundo Vencovsky (1987), para experimentos com duas ou três repetições, quanto mais próximo de 1 forem as relações $\mathrm{CVgi} / \mathrm{CVe}$, melhores as chances de ganhos com a aplicação de seleção em progênies, sugerindo que a característica pode ser trabalhada facilmente no melhoramento.

Porém, devido ao grande número de repetições utilizadas nos ensaios do presente trabalho, as estimativas obtidas foram suficientes para propiciar uma inferência precisa sobre o valor genotípico das progênies. A estimativa da acurácia seletiva foi superior a $66 \%$ para algumas características, em determinadas populações. Segundo Resende (2002), esses valores são desejáveis na fase inicial de programas de melhoramento genético.
Os coeficientes de variação experimental, para todas as características avaliadas, foram inferiores nas avaliações aos 6 meses, em relação às avaliações aos 3 meses. Para altura de plantas, houve uma variação de $10,8 \%$ a $24,7 \%$ (Tabela 1 ), para diâmetro de caule, a variação foi de $12,5 \%$ a $28,3 \%$ (Tabela 2), para projeção da copa, foi de $15,9 \%$ a $29 \%$ (Tabela 3), valores inferiores aos estimados para número de ramos, que variou de $25,5 \%$ a $36,7 \%$ (Tabela 4). Esses valores dos coeficientes de variação experimental podem ser atribuídos ao uso de parcelas de uma só planta, já que esses valores são estimados em função decrescente do número de árvores por parcela, conforme explicitado por Gomes (2000). No entanto, o uso de parcelas de uma planta permitiu a adoção de um grande número de repetições, fato que conduziu a herdabilidades ao nível de médias de progênies e acurácias de boas magnitudes. 
Tabela 1. Parâmetros genéticos para altura de plantas (em centímetros) de pinhão manso avaliadas aos 3 e 6 meses após o plantio, em cinco populações conduzidas na Fazenda Porteirão, da Biojan, em Janaúba, MG (abril de 2008 a janeiro de 2009).

\begin{tabular}{|c|c|c|c|c|c|c|}
\hline \multirow{2}{*}{ Parâmetros } & \multirow{2}{*}{ Meses } & \multicolumn{5}{|c|}{ Populações } \\
\hline & & 1 & 2 & 3 & 4 & 5 \\
\hline \multirow{2}{*}{$\mathrm{Va}$} & 3 & 19,0068 & 0,9809 & 1,7070 & 27,8026 & 6,8462 \\
\hline & 6 & 199,8341 & 28,9567 & 0,8025 & 65,0870 & 0,6426 \\
\hline \multirow{2}{*}{$\mathrm{Ve}$} & 3 & 144,0340 & 126,0501 & 158,5685 & 152,4445 & 255,0043 \\
\hline & 6 & 591,1526 & 658,1946 & 459,0258 & 515,0025 & 350,8461 \\
\hline \multirow{2}{*}{ Vf } & 3 & 163,0408 & 127,0310 & 160,2755 & 180,2471 & 261,8506 \\
\hline & 6 & 790,9867 & 687,1512 & 459,8283 & 580,0895 & 351,4886 \\
\hline \multirow{2}{*}{$h^{2} \mathrm{a}$} & 3 & 0,1166 & 0,0077 & 0,0106 & 0,1542 & 0,0261 \\
\hline & 6 & 0,2526 & 0,0421 & 0,0017 & 0,1122 & 0,0018 \\
\hline \multirow{2}{*}{$\mathrm{h}^{2} \mathrm{mp}$} & 3 & 0,5457 & 0,0718 & 0,0965 & 0,616025 & 0,2083 \\
\hline & 6 & 0,7295 & 0,2987 & 0,0172 & 0,5358 & 0,0180 \\
\hline \multirow{2}{*}{ acurácia } & 3 & 0,7387 & 0,2680 & 0,3106 & 0,7849 & 0,4564 \\
\hline & 6 & 0,8541 & 0,5465 & 0,1310 & 0,7320 & 0,1340 \\
\hline \multirow{2}{*}{ CVgi\% } & 3 & 7,9473 & 1,9015 & 2,1211 & 9,8860 & 3,6332 \\
\hline & 6 & 8,3323 & 3,2460 & 0,4787 & 4,7742 & 0,4621 \\
\hline \multirow{2}{*}{ CVgp \% } & 3 & 3,9736 & 0,9507 & 1,0605 & 4,9430 & 1,8166 \\
\hline & 6 & 4,1662 & 1,6230 & 0,2393 & 2,3871 & 0,2311 \\
\hline \multirow{2}{*}{$\mathrm{CVe} \%$} & 3 & 22,9346 & 21,6172 & 20,5251 & 24,6816 & 22,3956 \\
\hline & 6 & 16,0453 & 15,7291 & 11,4562 & 14,0516 & 10,8055 \\
\hline \multirow{2}{*}{$\mathrm{CVr}$} & 3 & 0,1733 & 0,0440 & 0,0517 & 0,2003 & 0,0811 \\
\hline & 6 & 0,2596 & 0,1032 & 0,0209 & 0,1698 & 0,0214 \\
\hline \multirow[t]{2}{*}{ Média geral } & 3 & $54,86 \mathrm{c}$ & $52,09 \mathrm{c}$ & $61,60 \mathrm{~b}$ & $53,37 \mathrm{c}$ & $72,02 \mathrm{a}$ \\
\hline & 6 & $169,66 \mathrm{~b}$ & $165,78 \mathrm{~b}$ & $187,14 \mathrm{a}$ & $168,98 \mathrm{~b}$ & $173,46 \mathrm{~b}$ \\
\hline \multirow{2}{*}{ DP } & 3 & 0,9334 & 0,5844 & 0,6652 & 1,0625 & 0,9065 \\
\hline & 6 & 2,6179 & 1,5571 & 1,0812 & 1,7431 & 0,9457 \\
\hline \multirow{2}{*}{ G \% } & 3 & 6,98 & - & - & 8,02 & - \\
\hline & 6 & 7,36 & - & - & 3,66 & - \\
\hline
\end{tabular}

Va: variância genética aditiva; Ve: variância residual; Vf: variância fenotípica individual; $h^{2} a$ : herdabilidade de plantas individuais; $h^{2} m p:$ herdabilidade da média de progênies; CVgi\%: coeficiente de variação genética aditiva individual; CVgp\%: coeficiente de variação genética entre progênies; $\mathrm{CVe} \%$ : coeficiente de variação residual; $\mathrm{CVr}=\mathrm{CVg} / \mathrm{CVe}$ : coeficiente de variação relativa; $\mathrm{M}$ : média geral; G: ganho genético predito na seleção dos dez melhores indivíduos em cada um dos experimentos, quando apresentavam acurácia superior a 66\%; DP: desvio padrão das médias de experimento. Médias seguidas por letras diferentes são estatisticamente diferentes. 
Tabela 2. Parâmetros genéticos para diâmetro de caule (em milímetros) de plantas de pinhão manso avaliadas aos 3 e 6 meses, em cinco populações conduzidas na Fazenda Porteirão, da Biojan, em Janaúba, MG (abril de 2008 a janeiro de 2009).

\begin{tabular}{|c|c|c|c|c|c|c|}
\hline \multirow{2}{*}{ Parâmetros } & \multirow{2}{*}{ Meses } & \multicolumn{5}{|c|}{ Populações } \\
\hline & & 1 & 2 & 3 & 4 & 5 \\
\hline \multirow{2}{*}{$\mathrm{Va}$} & 3 & 4,7729 & 2,3473 & 0,2327 & 1,0795 & 4,9639 \\
\hline & 6 & 29,8297 & 0,2114 & 1,3599 & 3,5506 & 9,8572 \\
\hline \multirow{2}{*}{$\mathrm{Ve}$} & 3 & 53,6524 & 51,3331 & 46,4263 & 45,8322 & 58,5105 \\
\hline & 6 & 130,2110 & 125,2832 & 65,6109 & 94,4807 & 70,0499 \\
\hline \multirow{2}{*}{$\mathrm{Vf}$} & 3 & 58,4253 & 53,6804 & 46,6590 & 46,9117 & 63,4744 \\
\hline & 6 & 160,0408 & 125,4947 & 66,9708 & 98,0313 & 79,9072 \\
\hline \multirow{2}{*}{$\mathrm{h}^{2} \mathrm{a}$} & 3 & 0,0817 & 0,0437 & 0,0050 & 0,0230 & 0,0782 \\
\hline & 6 & 0,1864 & 0,0017 & 0,0203 & 0,0362 & 0,1234 \\
\hline \multirow{2}{*}{$\mathrm{h}^{2} \mathrm{mp}$} & 3 & 0,4547 & 0,3066 & 0,0476 & 0,1879 & 0,4437 \\
\hline & 6 & 0,6616 & 0,0166 & 0,1695 & 0,2677 & 0,5600 \\
\hline \multirow{2}{*}{ acurácia } & 3 & 0,6743 & 0,5537 & 0,2181 & 0,4335 & 0,6661 \\
\hline & 6 & 0,8134 & 0,1287 & 0,4117 & 0,5174 & 0,7483 \\
\hline \multirow{2}{*}{ CVgi\% } & 3 & 8,1823 & 5,5579 & 1,5764 & 3,8248 & 6,3629 \\
\hline & 6 & 7,8301 & 0,6589 & 1,6161 & 2,7446 & 4,4479 \\
\hline \multirow{2}{*}{ CVgp\% } & 3 & 4,0911 & 2,7790 & 0,7882 & 1,9124 & 6,3629 \\
\hline & 6 & 3,9151 & 0,3295 & 0,8080 & 1,3723 & 4,4479 \\
\hline \multirow{2}{*}{$\mathrm{CVe} \%$} & 3 & 28,3338 & 26,4330 & 22,3083 & 25,1416 & 22,5297 \\
\hline & 6 & 17,7091 & 16,0517 & 11,3123 & 14,3560 & 12,4671 \\
\hline \multirow{2}{*}{$\mathrm{CVr}$} & 3 & 0,1444 & 0,1051 & 0,0353 & 0,0761 & 0,1412 \\
\hline & 6 & 0,2211 & 0,0205 & 0,0714 & 0,0956 & 0,1784 \\
\hline \multirow{2}{*}{ Média geral } & 3 & $26,7002 \mathrm{c}$ & $27,5661 \mathrm{c}$ & $30,6006 \mathrm{~b}$ & $27,1640 \mathrm{c}$ & $35,0151 \mathrm{a}$ \\
\hline & 6 & $69,7519 \mathrm{a}$ & $69,7750 \mathrm{a}$ & $72,1582 \mathrm{a}$ & $68,6552 \mathrm{a}$ & $70,5871 \mathrm{a}$ \\
\hline \multirow{2}{*}{ DP } & 3 & 0,5123 & 0,4377 & 0,3497 & 0,3790 & 0,5289 \\
\hline & 6 & 1,0621 & 0,5647 & 0,4479 & 0,5759 & 0,6636 \\
\hline \multirow{2}{*}{$\mathrm{G} \%$} & 3 & 7,00 & - & - & - & 5,03 \\
\hline & 6 & 6,35 & - & - & - & 5,61 \\
\hline
\end{tabular}

Va: variância genética aditiva; Ve: variância residual; Vf: variância fenotípica individual; $\mathrm{h}^{2} \mathrm{a}$ : herdabilidade de plantas individuais; $\mathrm{h}^{2} \mathrm{mp}$ : herdabilidade da média de progênies; CVgi\%: coeficiente de variação genética aditiva individual; CVgp\%: coeficiente de variação genética entre progênies; $\mathrm{CVe} \%$ : coeficiente de variação residual; $\mathrm{CVr}=\mathrm{CVg} / \mathrm{CVe}$ : coeficiente de variação relativa; M: média geral; G: ganho genético predito na seleção dos dez melhores indivíduos em cada um dos experimentos, quando apresentavam acurácia superior a 66\%; DP: desvio padrão das médias de experimento. Médias seguidas por letras diferentes são estatisticamente diferentes. 
Tabela 3. Parâmetros genéticos para número de ramos de plantas de pinhão manso avaliadas aos 3 e 6 meses, em cinco populações conduzidas na Fazenda Porteirão, da Biojan, em Janaúba, MG (abril de 2008 a janeiro de 2009).

\begin{tabular}{|c|c|c|c|c|c|c|}
\hline \multirow{2}{*}{ Parâmetros } & \multirow{2}{*}{ Meses } & \multicolumn{5}{|c|}{ Populações } \\
\hline & & 1 & 2 & 3 & 4 & 5 \\
\hline \multirow{2}{*}{$\mathrm{Va}$} & 3 & 0,0031 & 0,0318 & 0,0710 & 0,0987 & 0,0021 \\
\hline & 6 & 0,0019 & 0,0741 & 0,2382 & 0,0024 & 0,5811 \\
\hline \multirow{2}{*}{$\mathrm{Ve}$} & 3 & 1,3069 & 1,4064 & 1,0987 & 1,5068 & 1,2290 \\
\hline & 6 & 1,3000 & 1,3711 & 1,0324 & 1,1737 & 1,1956 \\
\hline \multirow{2}{*}{$\mathrm{Vf}$} & 3 & 1,3100 & 1,4383 & 1,1697 & 1,6055 & 1,2309 \\
\hline & 6 & 1,3019 & 1,4451 & 1,2706 & 1,1761 & 1,7767 \\
\hline \multirow{2}{*}{$h^{2} a$} & 3 & 0,0024 & 0,0221 & 0,0607 & 0,0615 & 0,0016 \\
\hline & 6 & 0,0015 & 0,0513 & 0,1875 & 0,0021 & 0,3271 \\
\hline \multirow{2}{*}{$h^{2} m p$} & 3 & 0,0233 & 0,1821 & 0,3812 & 0,3843 & 0,0157 \\
\hline & 6 & 0,0144 & 0,3418 & 0,6629 & 0,0201 & 0,7808 \\
\hline \multirow{2}{*}{ acurácia } & 3 & 0,1527 & 0,4267 & 0,6175 & 0,6199 & 0,1252 \\
\hline & 6 & 0,1201 & 0,5846 & 0,8142 & 0,1419 & 0,8836 \\
\hline \multirow{2}{*}{ CVgi\% } & 3 & 1,6370 & 5,4023 & 7,5934 & 9,1603 & 1,2657 \\
\hline & 6 & 1,0105 & 5,9511 & 11,6706 & 1,1563 & 16,4536 \\
\hline \multirow{2}{*}{ CVgp $\%$} & 3 & 0,8185 & 2,7011 & 3,7967 & 4,5802 & 0,6328 \\
\hline & 6 & 0,5052 & 2,9755 & 5,8353 & 0,5782 & 8,2268 \\
\hline \multirow{2}{*}{$\mathrm{CVe} \%$} & 3 & 33,5139 & 36,2100 & 30,5910 & 36,6674 & 31,7028 \\
\hline & 6 & 26,4118 & 26,1166 & 26,3157 & 25,5055 & 27,5682 \\
\hline \multirow{2}{*}{$\mathrm{CVr}$} & 3 & 0,0244 & 0,0746 & 0,1241 & 0,1249 & 0,0200 \\
\hline & 6 & 0,0191 & 0,1139 & 0,2217 & 0,0227 & 0,2984 \\
\hline \multirow[t]{2}{*}{ Média geral } & 3 & $3,4141 \mathrm{a}$ & $3,3028 \mathrm{a}$ & $3,5085 \mathrm{a}$ & $3,4289 \mathrm{a}$ & $3,4989 \mathrm{a}$ \\
\hline & 6 & $4,3192 \mathrm{a}$ & $4,5733 \mathrm{a}$ & $4,1818 \mathrm{a}$ & $4,2508 \mathrm{a}$ & $4,6331 \mathrm{a}$ \\
\hline \multirow{2}{*}{ DP } & 3 & 0,0577 & 0,0658 & 0,0681 & 0,0804 & 0,0559 \\
\hline & 6 & 0,0575 & 0,0729 & 0,0950 & 0,0546 & 0,1364 \\
\hline G\% & 6 & - & - & 14,60 & - & 25,05 \\
\hline
\end{tabular}

Va: variância genética aditiva; Ve: variância residual; Vf: variância fenotípica individual; $\mathrm{h}^{2} \mathrm{a}$ : herdabilidade de plantas individuais; $\mathrm{h}^{2} \mathrm{mp}$ : herdabilidade da média de progênies; CVgi\%: coeficiente de variação genética aditiva individual; CVgp\%: coeficiente de variação genética entre progênies; CVe\%: coeficiente de variação residual; $\mathrm{CVr}=\mathrm{CVg} / \mathrm{CVe}$ : coeficiente de variação relativa; $\mathrm{M}$ : média geral; G: ganho genético predito na seleção dos dez melhores indivíduos em cada um dos experimentos, quando apresentavam acurácia superior a 66\%; DP: desvio padrão das médias de experimento. Médias seguidas por letras diferentes são estatisticamente diferentes. 
Tabela 4. Parâmetros genéticos para projeção de copa (em centímetros) em plantas de pinhão manso avaliadas aos 3 e 6 meses, em cinco populações conduzidas na Fazenda Porteirão, da Biojan, em Janaúba, MG (abril de 2008 a janeiro de 2009).

\begin{tabular}{|c|c|c|c|c|c|c|}
\hline \multirow{2}{*}{ Parâmetros } & \multirow{2}{*}{ meses } & \multicolumn{5}{|c|}{ Populações } \\
\hline & & 1 & 2 & 3 & 4 & 5 \\
\hline \multirow{2}{*}{$\mathrm{Va}$} & 3 & 5,4018 & 0,7682 & 9,1540 & 30,2222 & 7,2026 \\
\hline & 6 & 69,6448 & 6,6043 & 2,2864 & 3,0012 & 8,0262 \\
\hline \multirow{2}{*}{$\mathrm{Ve}$} & 3 & 331,0773 & 289,1905 & 188,1307 & 179,6756 & 285,5204 \\
\hline & 6 & 977,6113 & 840,2859 & 686,4964 & 749,0285 & 947,4713 \\
\hline \multirow{2}{*}{ Vf } & 3 & 336,4792 & 289,9587 & 197,2848 & 209,8978 & 292,7230 \\
\hline & 6 & 1047,256 & 846,8903 & 688,7828 & 752,0296 & 955,4975 \\
\hline \multirow{2}{*}{$h^{2} a$} & 3 & 0,0160 & 0,0026 & 0,0464 & 0,1440 & 0,0246 \\
\hline & 6 & 0,0665 & 0,0078 & 0,0033 & 0,0040 & 0,0084 \\
\hline \multirow{2}{*}{$h^{2} m p$} & 3 & 0,1388 & 0,0258 & 0,3195 & 0,5990 & 0,1984 \\
\hline & 6 & 0,4034 & 0,0725 & 0,0322 & 0,0384 & 0,0776 \\
\hline \multirow{2}{*}{ acurácia } & 3 & 0,3726 & 0,1607 & 0,5652 & 0,7739 & 0,4455 \\
\hline & 6 & 0,6352 & 0,2692 & 0,1793 & 0,1960 & 0,2786 \\
\hline \multirow{2}{*}{ CVgi\% } & 3 & 3,6791 & 1,4497 & 4,3681 & 9,1061 & 3,3498 \\
\hline & 6 & 5,2455 & 1,5995 & 0,9166 & 1,1146 & 1,5041 \\
\hline \multirow{2}{*}{ CVgp $\%$} & 3 & 1,8396 & 0,7248 & 2,1841 & 4,5530 & 1,6749 \\
\hline & 6 & 2,6227 & 0,7998 & 0,4583 & 0,5573 & 0,7520 \\
\hline \multirow{2}{*}{$\mathrm{CVe} \%$} & 3 & 28,9789 & 28,1534 & 20,1605 & 23,5620 & 21,2896 \\
\hline & 6 & 20,1710 & 18,0953 & 15,9026 & 17,6349 & 16,3934 \\
\hline \multirow{2}{*}{$\mathrm{CVr}$} & 3 & 0,0635 & 0,1871 & 0,1083 & 0,1932 & 0,0787 \\
\hline & 6 & 0,1300 & 1,5314 & 0,0288 & 0,0316 & 0,0459 \\
\hline \multirow{2}{*}{ Média geral } & 3 & $63,1719 \mathrm{c}$ & $60,4635 \mathrm{c}$ & $69,2646 \mathrm{~b}$ & $60,3715 \mathrm{c}$ & $80,1165 \mathrm{a}$ \\
\hline & 6 & $159,0959 \mathrm{~b}$ & $160,6656 \mathrm{~b}$ & $164,9649 \mathrm{~b}$ & $155,4272 b$ & 188,3603 a \\
\hline \multirow{2}{*}{ DP } & 3 & 0,9865 & 0,8624 & 0,8464 & 1,1234 & 0,9526 \\
\hline & 6 & 2,0779 & 1,5094 & 1,3333 & 1,3976 & 1,6077 \\
\hline $\mathrm{G} \%$ & 3 & - & - & - & 9,08 & - \\
\hline
\end{tabular}

Va: variância genética aditiva; Ve: variância residual; Vf: variância fenotípica individual; $\mathrm{h}^{2} \mathrm{a}$ : herdabilidade de plantas individuais; $\mathrm{h}^{2} \mathrm{mp}$ : herdabilidade da média de progênies; $\mathrm{CVgi} \%$ : coeficiente de variação genética aditiva individual; $\mathrm{CVgp} \%$ : coeficiente de variação genética entre progênies; $\mathrm{CVe} \%$ : coeficiente de variação residual; $\mathrm{CVr}=\mathrm{CVg} / \mathrm{CVe}$ : coeficiente de variação relativa; $\mathrm{M}$ : média geral; G: ganho genético predito na seleção dos dez melhores indivíduos em cada um dos experimentos, quando apresentavam acurácia superior a 66\%; DP: desvio padrão das médias de experimento. Médias seguidas por letras diferentes são estatisticamente diferentes.

O coeficiente de variação genética expressa a magnitude da variação genética em relação à média do caráter. As estimativas foram variáveis para as características avaliadas, em função da população analisada. Porém, a característica que foi mais variável geneticamente foi o número de ramos aos 6 meses, nos experimentos 3 e 5, com CVgi de $12 \%$ e $16 \%$, respectivamente. Esses resultados concordam com os relatos de Maêda (2000).

A estimativa de herdabilidade em nível de indivíduo, de um modo geral, foi de baixa magnitude, para as características avaliadas em todas as populações, variando de $0,16 \%$ a $25,26 \%$. No entanto, a seleção individual pelo procedimento BLUP considera também as informações de parentes (informação das progênies), fato que propicia uma razoável acurácia seletiva. Em geral, herdabilidades individuais de baixas magnitudes são comuns para os caracteres quantitativos e, via de regra, conduzem a moderadas a altas magnitudes das herdabilidades em nível de médias de progênies (Resende, 2002).

Para a característica altura de plantas, houve diferença significativa entre as médias nas duas avaliações. Porém, as médias das populações 1 e 4 não diferiram 
significativamente e apresentaram considerável variabilidade genética, no qual o CVgi (\%) variou de $4,8 \%$ a $9,9 \%$ (Tabela 1 ). A herdabilidade individual para a mesma característica aos seis meses foi moderada ( $25 \%$ ) na população 1 e muito baixa ou nula nas outras populações, o que provavelmente se deve à natureza quantitativa e herança complexa da característica avaliada. Em experimentos com espécies florestais com menos de um ano de idade, os valores de herdabilidade para altura de plantas foram semelhantes aos estimados para pinhão manso, como em seringueira, em que a herdabilidade $\left(h_{a}^{2}\right)$ foi de $19 \%$ (COSTA et al., 2008) e de $22 \%$ (COSTA et al., 2006) e em erva-mate, de $5 \%$ (COSTA et al., 2005).

Houve herdabilidade da média de progênies nas populações 1 e 4, para altura de plantas aos 3 e 6 meses, com $\mathrm{h}^{2} \mathrm{mp}$ variando de $54 \%$ a $73 \%$, propiciando altas acurácias seletivas (73\% a $85 \%$ ), o que revela ótimas perspectivas para a seleção precoce nesses experimentos para altura de plantas (Tabela 1). Os valores de herdabilidade na média de progênies para altura de plantas foram semelhantes para seringueira com $51 \%$ (Costa et al., 2006) e erva-mate com 59\% (Costa et al., 2005).

Selecionando-se os dez melhores indivíduos para altura de plantas, pôde-se ter um ganho de 7,4\% na seleção aos 6 meses na população 1 e de $8 \%$ na 4 , aos 3 meses. Em erva-mate, o ganho genético com a seleção dos dez melhores indivíduos foi em torno de 4\% (Costa et al., 2005) e, em seringueira, os ganhos foram superiores, variando de $14 \%$ a $16 \%$. Nas outras populações, a altura de plantas não apresentou variabilidade suficiente para a prática da seleção precoce. A escolha do número igual a dez indivíduos não se baseou em uma intensidade de seleção desejada a priori. Mas sim, no número mínimo de indivíduos a serem incluídos em um pomar, de forma a permitir ganho máximo e evitar elevada taxa de endogamia.

Para a característica diâmetro de caule, as populações 1 e 5 apresentaram considerável variabilidade genética aos 3 e 6 meses, com CVgi (\%) variando de $4,4 \%$ a $8,2 \%$ e, na população 2 , aos três meses, com 5,6\% (Tabela 2). Esses resultados são condizentes com o relatado por Costa et al. (2006), em seringueira.

As estimativas de herdabilidade individual para diâmetro de caule foram moderadas aos 6 meses nas populações 1 e 5 (19\% e 12\%, respectivamente). Para essa característica, a herdabilidade nas outras populações foi praticamente nula. Esta característica também apresentou valores reduzidos de $h_{a}^{2}$ em erva-mate, $4 \%$ (Costa et al., 2005) e em seringueira, 2\% (Costa et al., 2006, 2008).

$\mathrm{Na}$ população 1, as estimativas indicam boa confiabilidade ou herdabilidade da média de progênies para diâmetro de caule (66\%), propiciando alta acurácia seletiva (81\%) na seleção individual aos 6 meses. A população 5 também apresentou boa acurácia seletiva para diâmetro de caule aos seis meses (75\%). Em espécies florestais, os valores de herdabilidade da média de progênies para diâmetro de caule foram um pouco inferiores: $41 \%$ para seringueira (41\%) (Costa et al., 2008) e 545 para erva-mate (Costa et al., 2005).

Desta forma, a seleção precoce para diâmetro de caule será eficiente nas populações 1 e 5, quando realizada aos 6 meses, trazendo ganhos genéticos de $6 \%$ e $5 \%$, respectivamente. Em erva-mate, Costa et al. (2005) estimaram ganhos genéticos próximos a 7\% na seleção dos melhores indivíduos. Não houve diferença significativa entre as médias de diâmetro do caule, aos 6 meses, em todos os experimentos, refletindo ausência de variabilidade entre as populações para essa variável.

A estimativa da herdabilidade individual para o número de ramos, aos 6 meses, foi moderada na população $5(33 \%)$ e muito baixa ou nula nas outras. Desta forma, a seleção aos 6 meses nas populações 3 e 5 trarão ganhos genéticos de $15 \%$ e $25 \%$, respectivamente. Nas outras populações, não é viável a seleção precoce para a variável número de ramos.

As médias para projeção de copa foram significativamente superiores na população 5 , nas duas avaliações, porém, dentro dessa população, não houve variabilidade genética suficiente para a prática da seleção precoce.

Apenas a população 4 apresentou variabilidade genética para projeção de copa, na avaliação aos 3 meses $(9 \%)$. Os valores de herdabilidade individual para projeção de copa foram baixos para todos os experimentos avaliados. Houve considerável confiabilidade ou herdabilidade na média de progênies para projeção de copa aos 3 meses $(60 \%)$, com acurácia de $77 \%$ para essa característica, o que revela boa perspectiva para a seleção, com ganhos genéticos preditos de $9 \%$. Nas outras populações, projeção de copa não teve variabilidade genética suficiente para realização da seleção precoce.

Apenas em uma das cinco populações avaliados, a característica projeção da copa exibiu variabilidade 
suficiente para permitir seleção precoce. Talvez seja uma característica de menor importância para avaliação de pinhão manso na fase vegetativa.

Após a avaliação de caracteres produtivos, será possível identificar quais populações possuem variabilidade genética suficiente para serem mantidas nos programas de melhoramento genético. E, adicionalmente, a seleção poderá ser implementada na prática, visando à obtenção de novas cultivares.

\section{Conclusões}

Houve variabilidade genética em quatro das cinco populações avaliadas, com estimativas de herdabilidade individuais de até $25,26 \%$ e herdabilidade de médias de progênies de até $78,08 \%$. Desta forma, é possível se obter ganhos genéticos consideráveis com a seleção dos dez melhores indivíduos para características vegetativas, variando de 3,6\% a 25,05\%, de acordo com a característica e a população avaliada, o que permite indicar ao melhorista quando (aos 3 ou 6 meses) e quais as melhores características a serem avaliadas. Os caracteres avaliados apresentam herança complexa, típica de características quantitativas.

\section{Agradecimentos}

Os autores agradecem aos Srs. Nagashi Tominaga e Jorge Kakida, pela parceria formada e pela condução dos experimentos.

\section{Referências}

ABREU, F. B.; RESENDE, M. D. V. de; ANSELMO, J. L.; SATURNINO, H. M.; BRENHA, J. A. M.; FREITAS, F.B. Variabilidade genética entre acessos de pinhão-manso na fase juvenil. Magistra, v. 21, p. 36-40, 2009.

ARRUDA, F.; BELTRÃO, N. E. M.; ANDRADE, A. P.; PEREIRA, W. E.; SEVERINO, L. S. Cultivo de Pinhão Manso (Jatropha curcas L.) como alternativa para o semi-árido nordestino. Revista Brasileira de Oleaginosas e Fibrosas, n. 8, p. 789-799, 2004.

BASHA, S.D.; SUJATHA, M. Genetic analysis of Jatropha species and interspecific hybrids between Jatropha curcas using nuclear and organelle specific markers. Euphytica, v. 168, n. 2, p. 197-214, 2009. Doi 10.1007/s10681-009-9900-0

BSBIOS. História do biodiesel no Brasil, 2008. Disponível em: $<$ http://www.bsbios.com/?menu=biodiesel $>$. Acesso em: 22 set. 2009.
CAVALCANTI, J. J. V.; RESENDE, M. D. V. de; CRISÓSTOMO, J. R.; PAIVA, J. R. Genetic control of quantitative traits and hybrid breeding strategies for cashew improvement. Crop Breeding and Applied Biotechnology, n. 7, p.186-195, 2007.

COSTA, R. B.; RESENDE, M. D. V. de; GONÇALVES, P. S. Prediction of genotypic values for yield in rubber tree clone test trials using REML/BLUP procedure. Crop Breeding and Applied Biotechnology, n. 2, p. 579-586, 2002a.

COSTA, R. B.; RESENDE, M. D. V. de; GONÇALVES, P. S.; SILVA, M. A. Individual multivariate REML/BLUP in the presence of genotype $\mathrm{x}$ environment interaction in rubber tree breeding. Crop Breeding and Applied Biotechnology, n. 2, p. 131-140, 2002b.

COSTA, R. B.; RESENDE, M. D. V. de; CONTINI, A. Z. REGO, F. L. H.; ROA, R. A. R. MARTINS, R. W. AVALIAÇÃO GENÉTICA DE INDIVÍDUOS DE ERVA-MATE (Ilex paraguariensis St. Hil.) NA REGIÃO DE CAARAPÓ, MS, PELO PROCEDIMENTO REML/BLUP. Ciência Florestal, Santa Maria, v. 15, n. 4, p. 371-376, 2005.

COSTA, R. B.; RESENDE, M. D. V.de; GONÇALVES, O. S.; REGO, F. L. H.; CONTINI, A. Z.; ROA, R. A. R.; MARTINS, W. J. Genetic evaluation of Hevea brasiliensis [(Willd ex Adr. De Juss.) Muell. Arg.] for juvenile vigour using the Reml/Blup method. Floresta \& Ambiente, n. 12, p. 08-14, 2006.

COSTA, R. B.; RESENDE, M. D. V. de; GONÇALVES, P. S.; CHICHORRO, J. F.; ROA, R. A. VARIABILIDADE GENÉTICA E SELEÇÃO PARA CARACTERES DE CRESCIMENTO DA SERINGUEIRA. Bragantia, Campinas, v. 67, n. 2, p. 299-305, 2008.

FARIAS NETO, J. T.; RESENDE, M. D. V. de. Aplicação da metodologia de modelos mistos (REML/BLUP) na estimação de componentes de variância e predição de valores genéticos em pupunheira (Bactris gasipaes). Revista Brasileira de Fruticultura, n. 23, p. 22-52, 2001.

FARIAS NETO, J. T.; OLIVEIRA, M. S. P.; MULLER, A. A.; NOGUEIRA, O. L.; ANAISSI, D. F. S. P. Variabilidade genética em progênies jovens de açaizeiro. Cerne, Lavras, v. 11, n. 4, p. 336-341, 2005.

GARCIA, C. H.; NOGUEIRA, M. C. S. Utilização da metodologia REML/BLUP na seleção de clones de eucalipto. Scientia Forestalis, n. 68, p.107-112, 2005.

GINWAL, H. S.; RAWAT, P. S.; SRIVASTAVA, R. L. Seed source variation in growth performance and oil yield of Jatropha curcas Linn. In Central India. Silvae Genetica, n. 53, p.186-192, 2004.

GINWAL, H. S.; PHARTYAL, S. S.; RAWAT, P. S.; SRIVASTAVA, R. L. Seed source variation in morphology, germination and seedling growth of Jatropha curcas Linn. In Central India. Silvae Genetica, n. 53, p. 76-80, 2005.

HELLER, J. Physic nut. Jatropha curcas L.: promoting the conservation and use of underutilized and neglected crops. Rome: International Plant Genetic Resources Institute, 1996. 66p.

KAUSHIK, N.; KUMAR, K.; KUMAR, S.; KAUSHIK, N.; ROY, $\mathrm{S}$. Genetic variability and divergence studies in seed traits and oil content of Jatropha (Jatropha curcas L.) accessions. Biomass and Bioenergy, n. 31, p. 479-502, 2007. 
MAÊDA, J. M. Avaliação de Parâmetros Genéticos e de Critérios de Seleção em Virola surinamensis (ROL) Warb. 2000. 94 p. il. Tese (Doutorado em Ciência Florestal), Universidade Federal de Viçosa, Minas Gerais.

MISSIO, R. F.; DIAS, L.A.S.; MORAES, M. L. T.; RESENDE, M. D. V. de. Selection of Pinus caribaea var. bahamensis progênies based on the predicted genetic value. Crop Breeding and Applied Biotechnology, n. 4, p. 399-407, 2004.

MISSIO, R. F.; SILVA, A. M.; DIAS, L. A. S.; MORAES, M. L. T.; RESENDE, M. D. V. de. Estimates of genetic parameters and prediction of additive genetic values in Pinus kesya progenies. Crop Breeding and Applied Biotechnology, n. 5, p. 394-401, 2005.

NOGUEIRA, O. L. ; GALVÃO, E. U. P. ; OLIVEIRA, R. P. de; MOREIRA, D. A. Relações entre caracteres fenotípicos quantitativos e a produção de palmito de açaizeiro (Euterpe oleracea Mart.). In: MOURÃO, L.; JARDIM, M. A.; GROSSMANN, M. Açaí: possibilidade e limites em processos de desenvolvimento sustentável no estuário amazônico. Belém: CEJUP, 2004. p. 27-36.

PAIVA, J. R.; CORDEIRO, E. R.; CORRÊA, M. C. M.; RESENDE, M. D. V. de. Acerola plant selection and breeding value prediction in second selection cycle progenies. Crop Breeding and Applied Biotechnology, n. 7, p. 125-132, 2007.

GOMES, P. F. Curso de estatística experimental. 14ed. Piracicaba: ESALQ, 2000. 477p.

RAO, G. R.; KORWAR, G. R.; SHANKER, A. K.; RAMAKRISHNA, Y. S. Genetic associations, variability and diversity in seed characters, growth, reproductive phenology and yield in Jatropha curcas (L.) accessions. Trees, n. 22, p. 697-709, 2008.

RESENDE, M. D. V. de. Análise estatística de modelos mistos via REML/BLUP na experimentaçao em melhoramento de plantas perenes. Colombo: Embrapa Florestas, 2000. 101 p. (Embrapa Florestas. Documentos, 47).
RESENDE, M. D. V. de. Genética biométrica e estatística no melhoramento de plantas perenes. Brasilia: Embrapa Informação Tecnológica: Colombo: Embrapa Florestas, 2002. 975 p.

RESENDE, M. D. V. de. Matemática e estatística na análise de experimentos e no melhoramento genético. Colombo: Embrapa Florestas, 2007. 561 p. 2007a.

RESENDE, M. D. V. de. SELEGEN-REML/BLUP: Sistema estatístico e seleção genética computadorizada via modelos lineares mistos. Colombo: Embrapa Florestas, 359 p., $2007 \mathrm{~b}$.

RESENDE, M. D. V. de; HIGA, A. R.; LAVORANTI, O. J. Predição de valores genéticos no melhoramento de Eucalyptus: melhor predição linear. In: CONGRESSO FLORESTAL PANAMERICANO, 1.; CONGRESSO FLORESTAL BRASILEIRO, 7., 1993, Curitiba. Floresta para o Desenvolvimento: Política, Ambiente, Tecnologia e Mercado: anais. São Paulo: SBS; [S.1.]: SBEF, 1993. v. 1, p. 144-147.

RESENDE, M. D. V. de; PRATES, D. F.; YAMADA, C. K., JESUS, A. de. Estimação de componentes de variância e predição de valores genéticos pelo método da máxima verossimilhança restrita (REML) e melhor predição linear não viciada (BLUP) em Pinus. Boletim de Pesquisa Florestal, Colombo, n. 32/33, p. 23 42, jan./dez. 1996.

ROCHA, M. G. B; PIRES, I. E.; XAVIER, A.; CRUZ, C. D.; ROCHA, R. B. Avaliação genética de progênies de meio-irmãos de Eucalyptus urophylla utilizando os procedimentos REML/ BLUP e E(QM). Ciência Florestal, Santa Maria, n.16, p. 369379, 2006.

SOUZA, A. G. C.; RESENDE, M. D. V. de; SILVA, S. E. L.; SOUZA, N. R. The cupuaçu genetic improvement program at Embrapa Amazônia Ocidental. Crop Breeding and Applied Biotechnology, n. 2, p. 471-478, 2002.

VENCOVSKY, R. Herança quantitativa. In: PATERNIANI, E.; VIEGAS, G. P. (Ed.). Melhoramento e produção do milho. 2. ed. Campinas: Fundação Cargill, 1987. p.137-214.

Recebido em 26 de agosto de 2009 e aprovado em 14 de junho de 2010 
